\title{
HIPERSENSIBILIDADE TUBERCULÍNICA EM CRIANÇAS MENORES DE UM ANO DE IDADE VACINADAS COM BCG ORAL *
}

\author{
Roberto Brólio** \\ Geraldo Chaves Salomon ** \\ Marília Belluomini ** \\ Péricles Alves Nogueira** \\ Stella Maria da Costa Nardy ***
}

RSPU-B/343

\begin{abstract}
Brólio, R. et al. Hipersensibilidade tuberculinica em crianças menores de um ano de idade vacinadas com $B C G$ oral. Rev. Saúde públ., S. Paulo, 11: $111-7,1977$
\end{abstract}

Resumo: São relatados os resultados da pesquisa da hipersensibilidade tuberculínica pelo PPD, Rt-23,2UT, em crianças menores de um ano de idade, vacinadas anteriormente com $B C G$ oral, pertencentes a uma área do municipio de São Paulo. Os resultados mostram que para 790 crianças houve $13,0 \%$ de reatores fracos e $16,3 \%$ de reatores fortes, com um total de $29,3 \%$ de reatores. São feitos comentários sobre a vacinação oral e o baixo percentual de reatores encontrados. no controle tuberculinico pós-vacinal, relativamente aos obtidos com o $B C G$ intradérmico.

Unitermos: Hipersensibilidade tuberculinica. Vacinação BCG. BCG por via oral.

\section{I $N$ T R O D U C $\bar{A}$ O}

Ninguém ignora o valor do teste tuberculínico para a identificação da infecção tuberculosa.

Realizado segundo uma técnica padronizada. o seu valor é inestimável quando aplicado em crianças não vacinadas pelo BCG, possibilitando fazer o levantamento dos coeficientes de infecção, que constituem indicadores de eleição, tanto na clínica como nas programaçōes sanitárias, pela sua fidelidade, sensibilidade e baixo custo.
Quando aplicado em crianças previamente vacinadas, revela a hipersensibilidade tuberculínica devida à impregnação do organismo pelos bacilos-vacina, sendo discutivel que haja uma relação entre hipersensibilidade tuberculínica e imunidade.

Com a generalização da prática da vacinação pelo $\mathrm{BCG}$, o teste tuberculínico vem perdendo o seu significado para o diagnóstico da infecção, porque não é possível distinguir a reatividade tuberculí-

* Trabalho realizado no Departamento de Epidemiologia da Faculdade de Saúde Pública da USP e no Centro de Saúde "Geraldo de Paula Souza", da mesma Faculdade.

* Do Departamento de Epidemiologia da Faculdade de Saúde Pública da USP - Av. Dr. Arnaldo, 715 - São Paulo, SP - Brasil.

** Do Centro de Saúde "Geraldo de Paula Souza" - Av. Dr. Arnaldo, 715 - São Paulo, SP - Brasil. 
BRólo, R. et al. - Hipersensibilidacle tuberculinica em crianças menores de um ano de idade vacinadas com BCG oral. Rer. Saúde públ., S. Paulo, 11:111-7, 1977.

nica da infecção natural por bacilos virulentos, daquela determinada pela infecção benígna, pelo BCG.

A infecção natural é a que resulta da implantação e desenvolvimento do bacilo de Koch no organismo. podendo ou não ser seguida de enfermidade. 0 risco de evolução patológica é maior nas crianças menores de 5 anos de idade e durante os dois primeiros anos que se seguem ao inicio da infecção.

A hipersensibilidade tuberculínica induzida pelo BCG está relacionada com vários fatores, entre os quais, a via de administração, não havendo contestação de que a via intradérmica produz maiores percentuais e mais elevada hipersensibilidade pós-vacinal do que a via oral.

$\mathrm{Na}$ prática corrente da aplicação do teste tuberculínico na infância, é de grande interesse saber se a criança foi ou não vacinada anteriormente e. se vacinada. qual a via de administração utilizada.

A pessoa vacinada pelo BCG intradérmico conserva a cicatriz vacinal no local da aplicação. Embora essa cicatriz seja idêntica à da vacina anti-variólica, localiza-se no braço direito, enquanto a $\mathrm{da}$ anti-variólica, no braço esquerdo, não havendo dificuldade para ser identificada.

Para as crianças vacinadas com BCG oral, essa identificação é feita pela informação dos adultos responsáveis pelas mesmas, sendo que durante o primeiro ano de vida as respostas são facilitadas, por se tratarem de acontecimento relativamente recente.

$O$ presente trabalho analisa os resultados da aplicação do teste tuberculínico em crianças menores de um ano de idade, vacinadas anteriormente com BCG oral, pertencentes a uma área do município de São Paulo, no período de 1970-1974.

\section{MATERIAL E METODOS}

A pesquisa da sensibilidade tuberculínica foi feita em crianças menores de um ano de idade, previamente vacinadas com BCG oral. atendidas no Serviço de Tisiologia do Centro de Saúde "Geraldo de Paula Souza" da Faculdade de Saúde Pública da Universidade de São Paulo (CS(TSP), no período de 1970 a 1974.

O CS-USP atende à população dos subdistritos de Vila Madalena e Jardim América da Capital de São Paulo.

As crianças examinadas pertencem às diferentes categorias econômico-sociais e étnicas de nossa população, e foram trazidas expontaneamente pelos adultos responsáveis pelas mesmas ou encaminhadas pelo Serviço de Higiene da Criança do CS-USP para o setor de diagnóstico do Serviço de Tisiologia da mesma Unidade Sanitária.

A vacina, administrada nas maternidades e Unidades Sanitárias de São Paulo, é fornecida pelo Instituto Butantan da Secretaria da Saúde do Estado, e apresentada na forma líquida, em frascos de 5 $\mathrm{ml}$. contendo $100 \mathrm{mg}$ de $\mathrm{BCG}$, conservada em geladeira entre 4 a $8^{\circ} \mathrm{C}$. para ser administrada por via oral.

Consoante a orientação da Divisão Nacional de Tuberculose (DNT) ${ }^{2}$. os recémnascidos recebem a conteúdo de um frasco (100 $\mathrm{mg}$ ) e as crianças com mais de 30 dias. até 4 anos de idade, recebem o conteúdo de dois frascos $(200 \mathrm{mg})$.

As crianças que fazem parte do presente estudo. em número de 790 . tomaram de uma a três doses de BCG (Tabela 1). A primeira dose foi dada na maternidade, depois do terceiro dia de vida, conforme as informaçōes obtidas. Para as crianças que tomaram mais de uma dose, as demais foram administradas no CS-USP, com intervalo de 15 a 30 dias. 
BROLIO, R. et al. - Hipersensibilidade tuberculinica em crianças menores de um ano de idade vacinadas com BCG oral. Rev. Saúde puibl., S. Paulo, 11:111-7, 1977.

O período de tempo decorrido entre a última dose da vacina administrada e a aplicação do teste tuberculínico foi sempre superior a dois meses, suficiente para que os bacilos-vacina pudessem produzir a respectiva impregnação do organismo.

A intradermo reação de Mantoux constou da aplicação de $0,1 \mathrm{ml}$ de tuberculina no terço inferior do antebraço esquerdo segundo a técnica recomendada pela DNT.

A tuberculina utilizada foi a de origem dinamarquesa, preparada pelo Serum Institute de Copenhague, diluída e distribuída pela DNT. É uma substância purificada (PPD), pertencente ao lote Rt-23, contendo 0,04 megr de tuberculina por 0,1 $\mathrm{ml}$, correspondendo a duas unidades de tuberculina (2UT).

A substância foi conservada em geladeira, ao abrigo da luz, e aplicada com seringas padronizadas, de vidro, com êmbolo de metal e agulhas de platina, fornecidas pela DNT.

$O$ critério de execução e leitura do teste foi sempre o mesmo, a cargo de pessoal previamente treinado por superviosoras qualificadas pela DNT.

A leitura do teste foi feita pela medida da induração tecidual, 72 horas depois da aplicação, e os resultados são distribuídos em três grupos: não reatores, quando há ausência de reação ou induração igual ou inferior a $4 \mathrm{~mm}$ de diâmetro transversal; reatores fracos, quando a induração é de 5 a $9 \mathrm{~mm}$ e, reatores fortes, quando a induração é igual ou superior a $10 \mathrm{~mm}$.

\section{R E S U L T A D O S}

Os resultados da aplicação do teste tuberculínico padronizado em 790 crianças menores de um ano de idade, previamente vacinadas com BCG administrado por via oral, podem ser vistos nas Tabelas 1 e 2 . $\mathrm{Na}$ Tabela 1 as crianças são distribuídas de acordo com o número de doses (uma ou mais doses) da vacina aplicada, e na Tabela 2, a distribuição é feita pelo número de crianças atendidas em cada ano, no período de 1970 a 1974.
Entre as 311 crianças que tomaram uma única dose de BCG houve $72,3 \%$ de não reatores, $11,9 \%$ de reatores fracos e $15,8 \%$ de reatores fortes, com um total de $27,7 \%$ de reatores.

Entre as 479 crianças que tomaram duas ou mais doses de BCG houve 69,5\% de não reatores, $13,8 \%$ de reatores fracos e $16,7 \%$ de reatores fortes, com um total de $30,5 \%$ de reatores.

A distribuição feita de acordo com o ano da aplicação do teste tuberculínico (Tabela 2) mostra um total de $29,4 \%$ de reatores, com os percentuais mais elevados nos anos de 1972 a 1974.

\section{DISCUSSAO E CONCLUSÃO}

A vacinação pelo BCG por via oral, administrada no Brasil desde 1927, vem sendo paulatinamente substituída pela via intradérmica a partir de 1970 , sem que se tenha feito uma avaliação, cientificamente aceita, de sua eficácia na imunização antituberculose.

Embora seja defendida por muitos autores $^{1,8}$, outros não manifestam o mesmo entusiasmo pela sua administração ${ }^{3,7}$.

A resposta positiva ao teste tuberculíni$\mathrm{co}$, do organismo previamente vacinado, não significa imunidade, mas que houve suficiente impregnação tissular pelos bacilos-vacina, embora Mackaness ${ }^{5,6}$ seja de opinião que existe uma certa correlação entre hipersensibilidade tuberculínica e imunidade, nas pessoas vacinadas.

Os dados apresentados mostram que o percentual do total de reatores é relativamente baixo, quando comparado ao obtido com a vacinação intradérmica, administrado com agulha e seringa, no mesmo grupo etário, em nosso meio, cujos testes tuberculínicos com PPD, Rt-23, 2UT, feitos 10 a 12 semanas depois da aplicação do $\mathrm{BCG}$, revelaram $31,1 \%$ de reatores fra$\cos$ e $53,6 \%$ de reatores fortes, com um total de $85,7 \%$ de reatores ${ }^{4}$. 
BROLIO, R. et al. - Hipersensibilidade tuberculinica em crianças menores de um ano de idade vacinadas com BCG oral. Rev. Saúde públ., S. Paulo, 11:111-7, 1977.

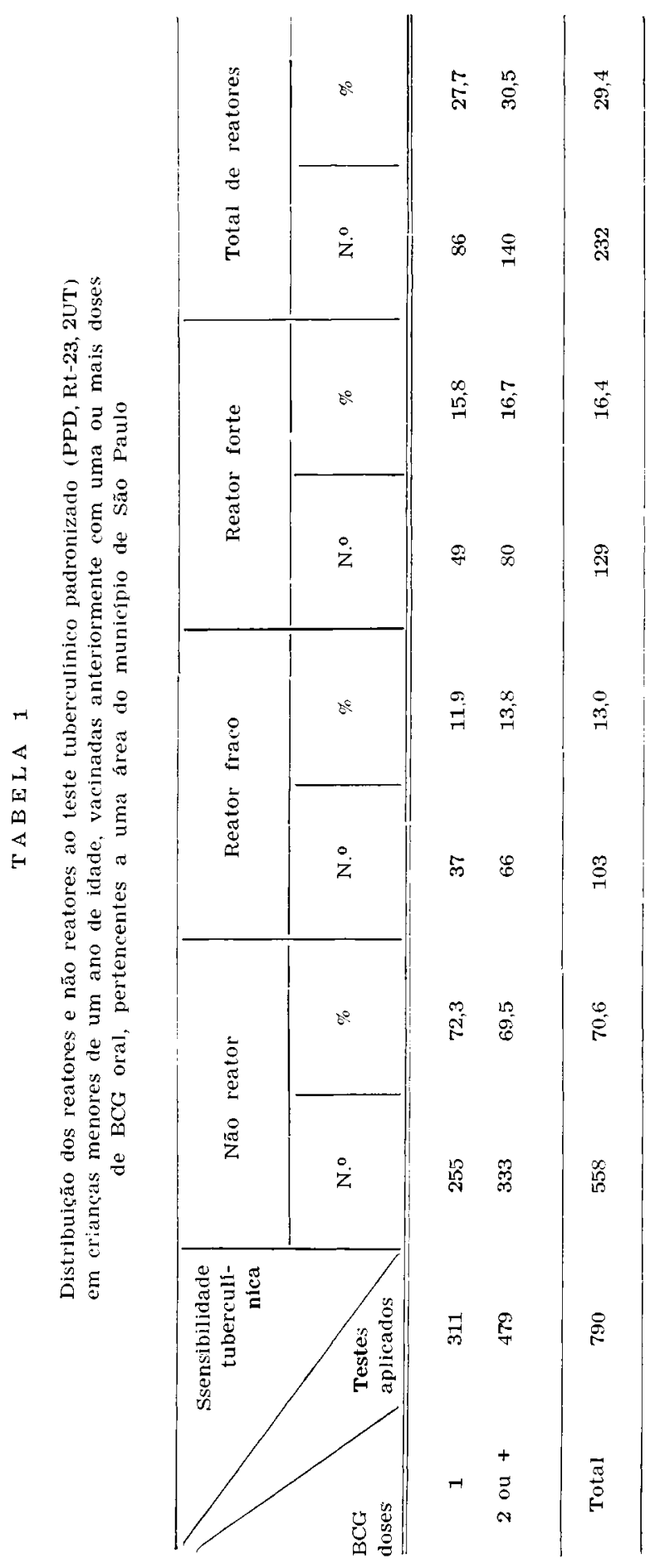


BROLIO, R. et al. - Hipersensibilidade tuberculinica em crianças menores de um $\varepsilon$ no de idade vacinadas com BCG oral. Rev. Saúde públ., S. Paulo, 11:111-7, 1977.

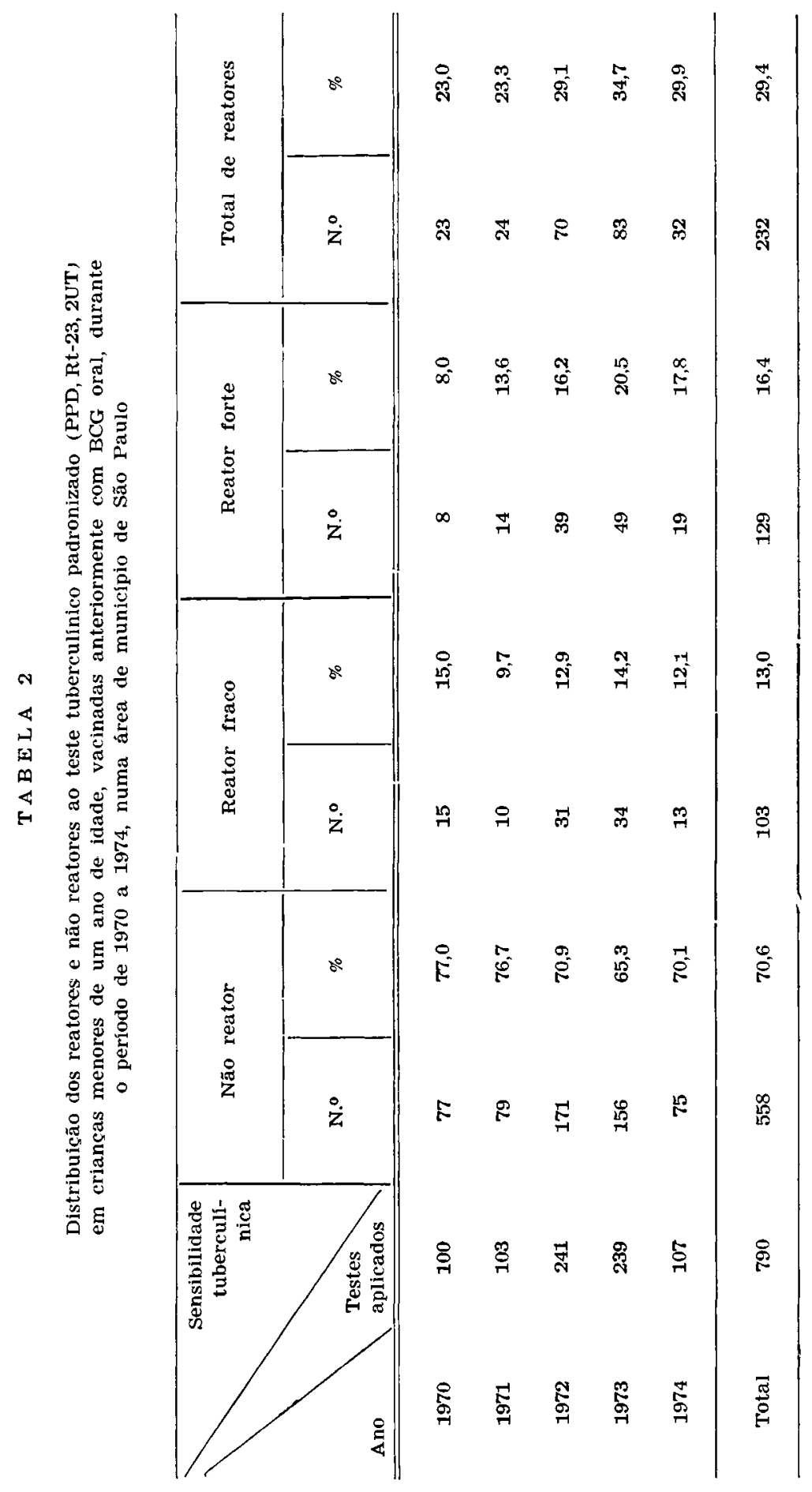


BROLIO, R. et al. - Hipersensibilidade tuberculinica em crianças menores de um ano de idade vacinadas com BCG oral. Rev. Saude públ., S. Paulo, 11:111 7, 1977.

Para autores brasileiros que têm trabalhado com BCG oral ${ }^{1,8}$ a hipersensibilidade tuberculínica pós-vacinal é fraca, mais freqüentemente demonstrável quando se fazem testes de Mantoux com 100 UT.

0 presente estudo mostra que o total de reatores é baixo, de $29,4 \%$, mas o percentual de reatores fortes é relativamente alto, de $16,4 \%$, superior ao dos reatores fracos, de $13,0 \%$.

Os resultados da Tabela 1 mostram que o percentual de reatores aumenta com a repetição das doses de BCG administra$\mathrm{do}$, indicando que a revacinação produz um reforço da hipersensibilidade tuberculínica, confirmando os trabalhos experi- mentais de Mackaness $^{6}$ que encontrou aumento de macrófagos ativos para o Mycobacterium tuberculosis quando o animal recebe repetidas doses de vacina.

Como a tuberculose congênita é muito rara e não tem sido descrita em nosso meio, presume-se que a hipersensibilidade tuberculínica das crianças vacinadas seja devida ao próprio BCG, administrado a partir do terceiro dia de vida, se bem que não se pode excluir a possibilidade de muitas dessas crianças terem sido igualmente infectadas por germes virulentos, pois a primo-infecção vacínica não isenta o organismo, exposto ao risco de contágios, de adquirir a infecção tuberculosa.

RSPU-B $/ 343$

BRóLIO, R. et al. [Tuberculinic hypersensibility among children under one year of life, vaccinated with BCG by oral administration.] Rev. Saúde públ., S. Paulo, 11:111-7, 1977.

ABSTRACTS: Hypersensibility reaction to tuberculin (PPD RT-23) was studied during five years among children under one year of age, vaccinated with $B C G$ by oral administration, living in an area of city S. Paulo, Brazil. Of 790 children, $13,0 \%$ showed a weak reaction and $16.4 \%$ showed a strong reaction, totalizing $29.4 \%$ reactors. Comparing the results of convertors after $B C G$ vaccination by intradermic administration, this paper shows that a low percentage of children became convertors after being vaccinated with $B C G$ by oral administration.

UNITERMS: Tuberculinic hipersensibility. $B C G$ vaccination. $B C G$, oral administration.

\section{REFERENCIAS BIBLIOGRAFICAS}

1. ASSIS, A. - Passado, presente e futuro da vacinação BCG. Rev. Serv, nac. Tubero., 8:7-30, 1964.

2. BRASIL. Divisão Nacional de Tuberculose. Ação antituberculose em nivel periférico. Instrucões técnicas. Rio de Janeiro, 1974.

3. BRollo, R. -- Duas epidemias de tu. berculose em criancas menores de três anos de idade, vacinadas com BCG oral, numa creche do município de São Paulo, Brasil. Rev. Saúde públ., S. Paulo, 8:283-96, 1974.

4. BROLIO, R. \& CARDOSO ALVES, H.A. - Análise dos resultados da vacinação de crianças pelo BCG intradermico com agulha e seringa e com o dermo-jet. Rev. Div. nac. Tuberc., 20:4-12, 1976 .

5. MACKANESS, G.B. - Cellular resistance to infection. $J$. exp. Med., 116:381406, 1962 . 
BROLIO, R. et al. - Hipersensibilidade tuberculínica em crianças menores de um ano de idade vacinadas com BCG oral. Rev. Saúde públ., S. Paulo, 11:111-7, 1977.

6. MACKANESS, G.B. - The immunological basis of acquired cellular resistance. J. exp. Med., 120:105-120, 1964.

7. PAUlA SOUZA, R. de - valor de oro. vacinação $B C G$ do recém-nascido. Rev. Div. nac. Tuberc., 17:61-71, 1973 .
8. ROSEMBERG, J. \& PASSOS FILHO, M.C da R. - Vacinação BCG pela técnica oral. Hospital, $\mathbf{R}$. de Janeiro, 78: 101-62, 1970.

Recebido para publicação em 08/09/1976 Aprovado para publicą̧ão em 10/09/1976 